\title{
La diffusion de l'appareil grammatical français : sources, vecteurs, remaniements (1860-1965)
}

\section{Gérard Vigner}

\section{(2) OpenEdition \\ Journals}

Édition électronique

URL: https://journals.openedition.org/dhfles/4449

DOI : $10.4000 /$ dhfles.4449

ISSN : 2221-4038

Éditeur

Société Internationale pour l'Histoire du Français Langue Étrangère ou Seconde

Édition imprimée

Date de publication : 1 décembre 2017

Pagination : 9-36

ISSN : 0992-7654

Référence électronique

Gérard Vigner, « La diffusion de l'appareil grammatical français : sources, vecteurs, remaniements

(1860-1965) ", Documents pour l'histoire du français langue étrangère ou seconde [En ligne], 58-59 | 2017, mis en ligne le 04 juin 2018, consulté le 27 mars 2023. URL : http://journals.openedition.org/dhfles/ 4449 ; DOl : https://doi.org/10.4000/dhfles.4449

Ce document a été généré automatiquement le 27 mars 2023.

Tous droits réservés 


\title{
La diffusion de l'appareil grammatical français : sources, vecteurs, remaniements (1860-1965)
}

\author{
Gérard Vigner
}

1 L'objectif de cette étude est d'examiner comment la France, à partir de la deuxième moitié du XIX ${ }^{e}$ siècle va mettre en place une offre de français à partir de ses ressources grammaticales et méthodologiques propres, et selon des réseaux de diffusion spécifiques, parallèlement aux enseignements du français mis en œuvre dans les pays étrangers par des enseignants et grammairiens étrangers.

2 Nous nous interrogerons à cette occasion sur les origines et l'évolution de ces répertoires grammaticaux entre 1860, simple repère sur lequel nous reviendrons, et 1964, au terme de la publication de l'ouvrage de G. Gougenheim et alii à propos du français fondamental $1^{\text {er }}$ degré.

3 Nulle volonté d'exhaustivité, mais des prises d'échantillons destinées à poser les bases d'un parcours qui mériterait certainement d'être repris dans le détail ; une tentative pour situer des choix en matière de descriptions grammaticales qui sont en même temps des choix institutionnels ou des choix par défaut, nous le verrons.

\section{Une volonté nouvelle}

4 Nulle date, nul décisionnaire identifié pour situer la naissance de ce vaste mouvement par lequel la France, comme État-nation, va décider d'entrer plus ou moins officiellement dans le jeu d'une diffusion de la langue française hors de France. Mais une période qui s'étale quasiment sur un demi-siècle (de 1850 à 1900) et, à l'origine de ce mouvement, les acteurs les plus divers, membres du clergé, intellectuels et hommes politiques de culture juive, universitaires et hommes politiques de sensibilité plus ou moins laïque, sans concertation particulière entre eux, mais animés par une égale volonté de se projeter hors de France pour porter les messages les plus variés dont l'usage et l'enseignement de la langue française seront le commun vecteur, une volonté 
de faire rayonner la France par la diffusion de sa langue et d'un certain nombre de valeurs associéesi. On se gardera cependant, risque d'une illusion rétrospective, de mettre dans une même visée idéologique, des instances aussi variées que l'œeuvre des écoles d'Orient, - soucieuse de porter le message évangélique en Orient (dans les pays du Levant) par l'ouverture d'écoles ${ }^{1}-$, l'Alliance israélite universelle qui vise à émanciper et "régénérer " les communautés juives d'Orient opprimées - alors que l'Alliance française, créée une vingtaine d'années plus tard, dans un contexte politique différent dans lequel la rivalité avec l'Allemagne va occuper une place importante, vise à diffuser un message et une influence politique fondés sur le principe d'une conquête morale, constamment affirmée dans les colonies, et la diffusion de valeurs de civilisation auprès de publics européens ou nord-américains. Quant à la Mission laïque française, créée en 1902, elle l'est par un certain nombre de personnes et de mouvements - Ligue française de l'Enseignement, loges franc-maçonniques notamment - qui considèrent que l'Alliance française fait preuve de beaucoup trop de complaisance à l'égard des écoles congréganistes ${ }^{2}$. Ce qui n'empêchera pas d'ailleurs le même Pierre Foncin de devenir le premier président de la MLF (voir Vigner 2015b), en même temps qu'il restait secrétaire général de l'AF. Un très large mouvement se dessine donc, dans la diversité des motivations de ses différents acteurs, mais dont la référence à la langue française constituera un point d'action partagé. Des institutions privées certes, mais pour certaines, l'AF ou la MLF, créées à l'initiative de hauts-fonctionnaires et d'universitaires. L'État français n'est pas loin, il observe d'abord, mais progressivement par des biais variés va apporter son appui à ces différents organismes.

\section{Un outil de référence : la deuxième grammaire scolaire}

Pour paradoxal que cela puisse paraître, lorsqu'il fut question de diffuser la langue française hors de France, les institutions dont nous venons de parler ne disposent d'aucun outil d'enseignement approprié de la langue, notamment sous la forme d'une grammaire descriptive du français, adaptée aux besoins de publics non francophones. Les décideurs, les responsables d'établissement, les enseignants eux-mêmes, devront se reporter aux manuels de grammaire en usage en France et élaborer, dans des conditions très empiriques, une pédagogie du français qui tout à la fois s'inspirera de principes venus de la méthode directe et de descriptions du français proposées dans le cadre de la deuxième grammaire scolaire, c'est-à-dire d'une grammaire destinée aux publics scolaires français.

6 Nous devons à André Chervel cette dénomination $(1977,2006)$ qui sert, selon lui, à désigner un mouvement grammatical qui entre en rupture avec la grammaire de Noël et Chapsal, grammaire qui occupera l'essentiel du marché scolaire entre 1815 et 1850, dérivée de la grammaire générale elle-même mise en œuvre dans les écoles centrales de la Révolution. Grammaire hautement abstraite qui demandait aux élèves derrière chaque phrase observée de retrouver par reformulations diverses un certain nombre de schémas fondamentaux de la langue.

7 L'outil fut cependant très rapidement critiqué par les praticiens, comme par les inspecteurs qui commençaient à circuler dans les écoles et sans qu'il soit possible d'assigner ce mouvement à une origine particulière. Les enseignants, auteurs de manuels, éditeurs, s'orientèrent progressivement vers des choix différents, plus 
proches des besoins des élèves, une grammaire des fonctions, susceptible d'offrir à l'enseignement de l'orthographe les explications dont ces derniers avaient grandement besoin. On observe la langue, on l'analyse (analyse grammaticale et analyse logique), on nomme les constituants de la langue par le moyen d'un métalangage que l'on va progressivement stabiliser et homogénéiser. La publication de la nomenclature de $1910^{3}$ constituera le point d'aboutissement d'un mouvement qui sera beaucoup plus le fait de praticiens de l'enseignement que résultant de propositions issues des travaux des grammaires savantes.

Ce courant grammatical, et pédagogique, coïncidant avec la création de l'école républicaine dans le début des années 1880 , va donner lieu à un mouvement éditorial d'une ampleur exceptionnelle, production éditoriale qui doit aussi être interprétée sur un plan politique. Il s'agit de favoriser l'unité du pays à partir de la référence à une langue commune, grammaire qui en même temps a pour objectif de donner à tous les petits Français la maittrise d'une orthographe particulièrement complexe.

9 Mais dans le même temps, ce formidable engagement dans la diffusion de la langue française à l'étranger ne va pas se traduire par la mise au point d'un outil de référence approprié. On peut citer cependant l'ouvrage de L. Clédat, son Précis d'orthographe et de grammaire phonétiques pour l'enseignement du français à l'étranger (Masson 1890), qui s'inscrit dans le très large mouvement de renouvellement des travaux portant sur la phonétique et sur l'enseignement de la prononciation.

Cette absence est-elle l'expression d'une vision indifférenciée des publics, étrangers européens, enfants indigènes des colonies, petits français patoisant, ce qui n'est pas complètement impossible, ou bien la difficulté dans laquelle se trouvent les acteurs de ce mouvement à concevoir un outillage descriptif spécifique?

11 Nous examinerons, à partir des sources disponibles, les premiers choix entrepris par les institutions évoquées plus haut. ${ }^{4}$

\section{L'Alliance israélite universelle}

12 Nous ne reviendrons pas ici sur le cadre et les conditions de création de l'Alliance israélite universelle (voir Kaspi 2010; Spaëth 2001). Fondée en 1860 par un groupe d'intellectuels et d'hommes politiques français de confession juive, l'AIU se donne pour projet d'apporter son appui aux communautés juives d'Orient, communautés souvent persécutées et dans tous les cas marginalisées, en ouvrant partout où cela sera possible des écoles qui apporteront un savoir nouveau et contribueront à la « régénération » de ces populations. La première école sera ouverte à Tétouan au Maroc en 1862 et de très nombreuses suivront jusque dans la lointaine Perse.

13 L'AIU va choisir de fonder son action éducative sur la diffusion et l'usage de la langue française. Choix qui ne sera pas exclusif, puisque l'AIU veillera à ce que, selon les pays, d'autres langues soient enseignées. Mais dans tous les cas, la langue française y occupera une place privilégiée. Dans ses Instructions générales pour les professeurs (1903: 38), l'AIU rappelle en effet :

Le but de l'école primaire n'est pas d'enseigner une langue pour elle-même, [...] la langue est non pas le but, mais l'instrument nécessaire pour atteindre un but qui est l'éducation générale de l'enfant. Une langue occidentale est indispensable pour cet objet, puisqu'elle est l'unique moyen de mettre l'enfant en communication et en contact avec la civilisation occidentale. 
L'enseignement de la langue française a donc uniquement pour but de mettre l'enfant en possession d'une langue au moyen de laquelle il puisse être instruit, éclairé, et continuer à son tour son éducation intellectuelle lorsqu'il sera sorti de l'école.

Cet enseignement sera donc surtout pratique. Il visera avant tout à obtenir ce résultat : que l'enfant comprenne facilement la langue française parlée ou écrite et lise aisément les auteurs français. grammaticaux à assimiler, mais sur des compétences à acquérir, dont l'outil grammatical sera un des vecteurs privilégiés. Un peu plus loin (p. 39), il est précisé encore :

Dans aucun cas, l'enseignement de la grammaire française ne doit être un enseignement mort, un simple exercice de mémoire, il sera surtout un enseignement oral. Le livre de grammaire peut faciliter à titre d'auxiliaire la tâche du maître, mais à condition qu'il ne jouera qu'un rôle secondaire et qu'il serve à faciliter la tâche du maître et non à le remplacer. L'enfant doit s'en servir principalement pour apprendre les conjugaisons des verbes réguliers et irréguliers, les adjectifs possessifs et démonstratifs, les pronoms, quelques séries de noms, d'adjectifs et de verbes irréguliers, et pour les exercices écrits dont la plupart de ces livres sont accompagnés. Presque tout le reste doit être enseigné de vive voix.

On trouve ici un corps de recommandations, qui témoignent d'une défiance incontestable à l'égard d'un enseignement qui ne serait qu'un enseignement de pur savoir et sur des objectifs de simple reconnaissance et de dénomination des formes ${ }^{5}$.

Pour autant, cette volonté incontestable de modernisation de l'enseignement du français s'appuie sur un descriptif grammatical issu des grammaires en usage dans les écoles de France :

Classes 4 et 3. -1 et 2 . Nom et article. - 3. Adjectif. - 4. Pronom. - 5. Verbes auxiliaires. -6 Verbes réguliers. -7 . Verbes irréguliers. -8 . Prépositions régies par les verbes. -9 et 10 . Adverbes, conjonctions, interjections. La proposition, signes de ponctuation.

Classes 2 et 1 . - 1 et 2 . Noms, noms composés, quelques difficultés simples sur le genre et le nombre de certains mots. -3 et 4 . Adjectifs, règles élémentaires sur : même, tout, quelque, etc., adjectifs numéraux. $-5,6$, 7. Pronoms, verbes, participes. - 8. Prépositions adverbes, conjonctions, interjections. - 9. Proposition, ponctuation. - 10. Révision.

S'il convient donc, selon les auteurs du texte, de se défier des ouvrages de grammaires dans leur forme littérale, en revanche le répertoire grammatical proposé est des plus classiques, on y trouve les parties du discours déjà inventoriées par Lhomond en 1784 et tend à privilégier une approche analytique de la langue.

L'ouvrage de Moïse Fresco ${ }^{6}$, publié en 1895, et s'adressant aux élèves des écoles d'Orient et d'Afrique du nord, s'organise en une suite d'exercices portant sur les accords de genre et de nombre, sur la conjugaison qui, dans le choix des formes de référence, serait parfaitement à sa place dans une école française. 


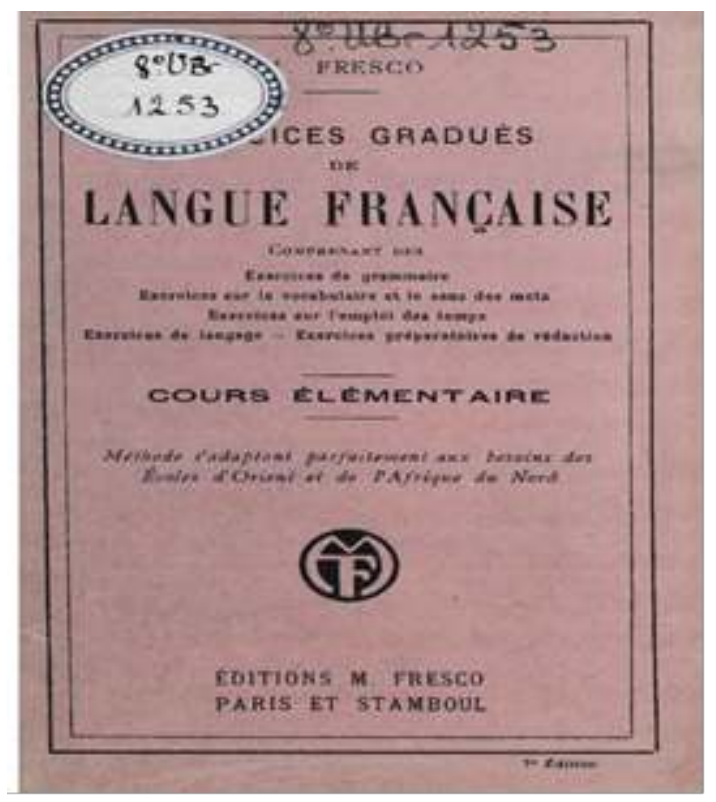

\section{L'Alliance française}

Créée en 1883, l'Alliance française se définit ainsi: «L'association nationale pour la propagation de la langue française dans les colonies et à l'étranger ». Nous ne reviendrons pas ici sur les conditions de création de cette nouvelle institution. Nombreux sont les travaux consacrés à sa création et à son fonctionnement (Chaubet 2006, Cortier 1998, Nishiyama, 2001, 2005). L'Alliance se consacre ainsi à la diffusion de l'enseignement du français dans les colonies et appuiera les écoles ouvertes dans les pays du Levant, pour l'essentiel des écoles non-laïques (congréganistes, grecques orthodoxes, américaines, israélites et laïques). Même si l'AF ne met pas en place de programme de français spécifique ou un outillage grammatical commun à toutes les écoles, elle va contribuer cependant par son action à diffuser un appareil grammatical et une nomenclature issus des usages français. Deux grands vecteurs :

les manuels de grammaire diffusés dans les écoles pris dans les listes de manuels publiés en France ${ }^{7}$

les cours de vacances ouvert à partir de 1894 et que Ferdinand Brunot va rapidement prendre en charge.

\section{La collection de manuels de grammaire « Larive et Fleury »}

Deux mots peut-être de cet éditeur scolaire, Armand Colin (1842-1900) qui entre au conseil d'administration de l'AF dès $1883^{8}$. Personnage discret, mais homme d'influence, il se lance en 1871 dans l'édition scolaire à destination de l'enseignement primaire ${ }^{9}$. Entre 1872 et 1889, il couvre environ le quart du marché du livre scolaire, 50 millions de volumes vendus environ et la grammaire de Larive et Fleury ${ }^{10}$, pour la même période, correspond à une vente de 12 millions d'exemplaires environ ${ }^{11}$. Armand Colin édite aussi les «instituteurs de la nation $»^{12}$, Ernest Lavisse (1842-1922) pour l'histoire, Pierre Foncin (1841-1916), pour la géographie, Paul Vidal de la Blache 
(1845-1918) pour son atlas et Paul Bert (1833-1886) pour les sciences. Un catalogue riche, soigneusement construit, pour des ouvrages conçus avec le plus grand soin ${ }^{13}$. acheté par les écoles ouvertes notamment dans les pays du Levant. On sait que l'AF,
dans les premières années, s'investit particulièrement dans les écoles, domaine qu'elle
abandonnera progressivement dans les débuts du XX $\mathrm{XX}^{\mathrm{e}}$ siècle pour s'orienter plutôt vers les cours d'adulte.

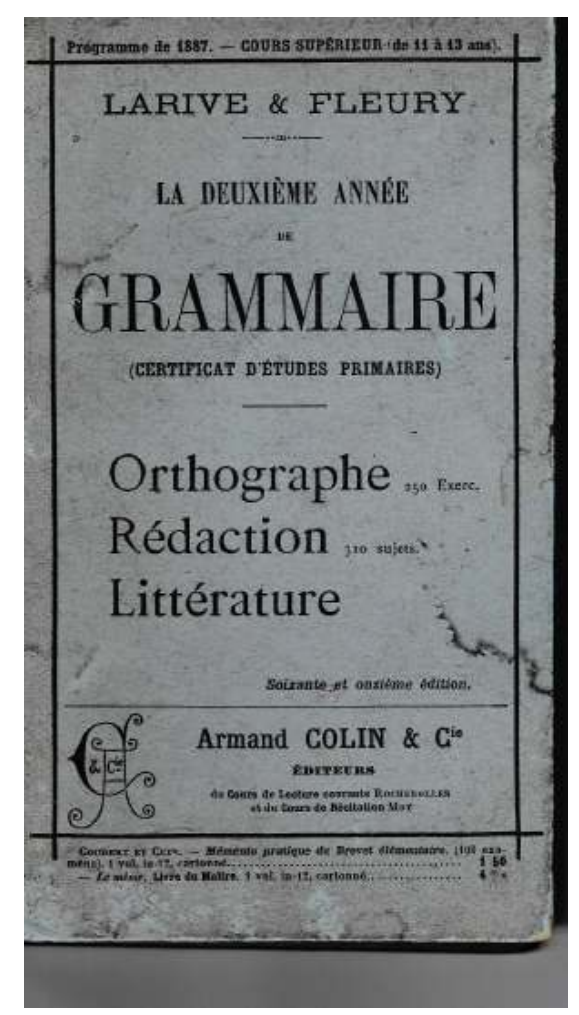

Lors d'une visite dans l'école des Sœurs de Sophia (sic) en Bulgarie, le délégué de l'Alliance française, Ferdinand Dreyfus, note ceci :

Nous jetons un coup d'œil sur les cahiers et sur les livres. Nous retrouvons les mêmes manuels que dans nos écoles primaires, Le Tour de la France par deux enfants et nous saluons nos deux amis, Larive et Fleury. ${ }^{14}$

Remarque significative qui fait clairement apparaître l'importance de l'aire de diffusion de cette collection et son influence dans l'enseignement du français. Si très nombreux sont les travaux consacrés aujourd'hui aux écoles françaises dans les pays du Levant, rares sont ceux qui vont jusqu'à inventorier (mais dispose-t-on des archives à cet effet ?) les manuels commandés par les écoles et en usage dans les classes ${ }^{15}$. Or on sait combien les manuels ont un effet modélisant sur la façon qu'ont les maîtres et les maîtresses de penser et d'organiser les apprentissages.

\section{Les cours de vacances}

Les cours sont créés suite à une décision du Conseil d'administration, prise le 4 décembre 1893 : «cours qui seront réservés aux étrangers désireux de perfectionner 
leurs connaissances littéraires et grammaticales» (BAF 1893: 47). Ces cours comprendront une composante grammaticale, que l'on distinguera en cours élémentaire et cours supérieur. Ferdinand Brunot prendra en charge le cours supérieur et fera partie du comité de patronage de ces cours qui connaîtront très tôt un grand succès : une cinquante d'auditeurs pour la première session, celle de l'été 1894, mais 460 environ en 1896 et 510 en 1897. Les cours à Paris seront d'ailleurs dédoublés et d'autre vont ouvrir à Nancy, puis à Caen.

Le programme de ces cours, qui vont d'adresser à un vaste public d'enseignants étrangers, s'inscrit dans les catégories descriptives d'une grammaire que nous pouvons qualifier aujourd'hui de traditionnelle, mais qui en ce temps-là s'inscrivait dans les perspectives tracées par la deuxième grammaire scolaire :

\begin{tabular}{|c|c|c|c|c|c|}
\hline 1895 & & 1896 & & 1897 & \\
\hline $\begin{array}{l}\text { Cours } \\
\text { élémentaire }\end{array}$ & Cours supérieur & $\begin{array}{l}\text { Cours } \\
\text { élémentaire }\end{array}$ & Cours supérieur & $\begin{array}{l}\text { Cours } \\
\text { élémentaire }\end{array}$ & Cours supérieur \\
\hline $\begin{array}{l}\text { Valeur et } \\
\text { emploi des } \\
\text { auxiliaires } \\
\text { Les temps du } \\
\text { passif } \\
\text { Les divers } \\
\text { temps du } \\
\text { passé au mode } \\
\text { indicatif } \\
\text { Le subjonctif } \\
\text { Le } \\
\text { conditionnel } \\
\text { La } \\
\text { concordance } \\
\text { des temps } \\
\text { Explication } \\
\text { Grammaticale } \\
\text { de textes } \\
\text { empruntés } \\
\text { aux auteurs } \\
\text { du XIXe siècle } \\
\text { Exercices } \\
\text { d'application } \\
\text { sur le cours } \\
\text { théorique }\end{array}$ & 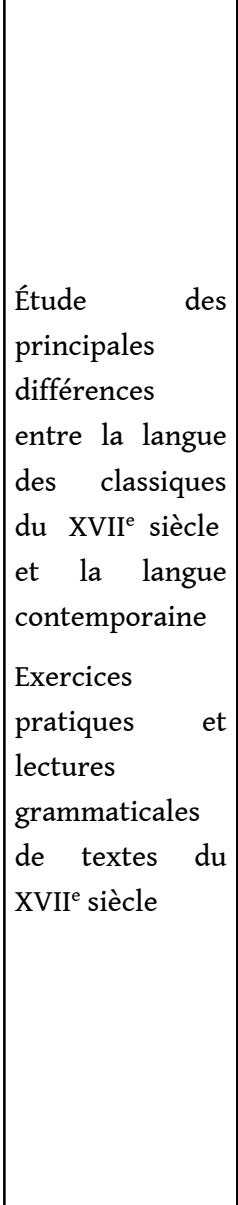 & $\begin{array}{l}\text { Syntaxe de } \\
\text { l'article } \\
\text { Syntaxe du } \\
\text { pronom } \\
\text { Explication } \\
\text { grammaticale } \\
\text { de textes }\end{array}$ & $\begin{array}{l}\text { Étude comparée } \\
\text { des formes et de } \\
\text { la syntaxe des } \\
\text { temps des verbes } \\
\text { dans le français } \\
\text { du XVII et du } \\
\text { XIX siècles } \\
\text { Les auxiliaires } \\
\text { être et avoir } \\
\text { Temps } \\
\text { périphrastiques } \\
\text { formés avec } \\
\text { aller, s'en aller } \\
\text { Les temps du } \\
\text { passif français } \\
\text { Le passé simple } \\
\text { et le passé } \\
\text { composé } \\
\text { Concordance des } \\
\text { temps dans les } \\
\text { phrases } \\
\text { coordonnées et } \\
\text { subordonnées }\end{array}$ & & $\begin{array}{l}\text { Observations } \\
\text { générales } \\
\text { Subjonctif et } \\
\text { conditionnel } \\
\text { Subjonctif et } \\
\text { indicatif dans } \\
\text { les propositions } \\
\text { complétives } \\
\text { Subjonctif et } \\
\text { indicatif dans } \\
\text { les propositions } \\
\text { causales, } \\
\text { consécutives, } \\
\text { hypothétiques, } \\
\text { concessives, } \\
\text { comparatives, } \\
\text { temporelles }\end{array}$ \\
\hline
\end{tabular}

On peut donc penser que ce très important public des cours de vacances va être imprégné d'une terminologie - que l'on retrouvera d'ailleurs dans la nomenclature de 1910 -, et qui sera de la sorte très largement diffusée à l'étranger. 


\section{Dans les colonies}

Si le ministère de l'Instruction publique pour la France dispose de programmes nationaux, et, à partir de 1910, propose une nomenclature officielle pour la grammaire, rien de tel en revanche pour ce qui est du français hors de France. Nulle instance centralisatrice, nul répertoire plus ou moins officiel. Chacun des acteurs dans son territoire d'intervention, dans ses réseaux propres, opère les choix qui lui paraissent les plus appropriés par rapport à un cadre grammatical considéré comme légitime, chacun cependant s'efforçant de mettre en place des programmes en référence aux usages français.

31 Une organisation de l'école indigène se met en place dans chacune des grandes aires territoriales des colonies, dans un environnement institutionnel et politique différencié. Avant même que des programmes soient mis en place, des auteurs, tels Louis Machuel ou Irénée Carré diffusent des méthodes de langage pour enseigner le français dans les colonies.

Cette chronologie correspond aux étapes de contrôle des territoires et de mise en cohérence des politiques éducatives: 1890 pour l'Algérie (Vigner 2015a), pour la Tunisie, notamment 1909, avec la mise en place d'un programme général, 1920 pour le Maroc, 1914 pour l'AOF, 1917 pour l'Indochine avec le Règlement général de l'Instruction publique.

33 Un lien très fort est établi entre la méthode Carré et l'enseignement du français dans les colonies. Un examen rapide de certains programmes fait clairement apparaître une certaine homogénéité des choix en matière terminologique, qu'il s'agisse des contenus de langue devant trouver place dans les activités de langage ou d'un enseignement explicite d'un certain nombre de catégories grammaticales. Les programmes de 1924 pour l'AOF feront ainsi explicitement références aux 10 catégories du discours ${ }^{16}$.

\section{L'entre-deux guerres}

Période paradoxale en ce sens que la réorganisation du monde qui, notamment en Europe, résulte de l'effondrement des empires centraux et des différents traités de paix signés entre 1919 et 1922, n'a aucune traduction en matière d'innovation méthodologique et d'évolution nette dans le choix des références grammaticales. La France se soucie certes de développer son réseau d'instituts français dans les pays d'Europe centrale nouvellement créés à l'initiative notamment de Ferdinand Brunot, 4 instituts le sont en 1914, 29 en 1933 (voir Chevalier 2001, 2010), actions relayées sur place par des universitaires tels L. Tesnière ou A. Meillet. De nombreuses missions universitaires sont organisées dans les pays d'Europe centrale.

De même dans les colonies, sont désormais installées dans tous les territoires, des écoles indigènes, selon la doctrine du moment en vigueur, de dissociation des réseaux de formation entre réseaux européens et réseaux indigènes, avec à chaque fois le souci de proposer un enseignement adapté ${ }^{17}$. Le Maroc, récemment intégré dans l'empire à partir de 1912, publie ses premiers programmes en 1920 : Circulaire du 30 août 1920. Plan d'études et programme de l'enseignement indigène.

La France ouvre cependant un institut spécifiquement dédié à la formation des professeurs de français devant exercer à l'étranger « L'école de préparation des professeurs 
de français à l'étranger", à la Sorbonne en 1920, qui d'une certaine manière est le pendant institutionnel des cours de vacances de l'AF auxquels Brunot s'était déjà consacré $^{18}$.

L'Alliance française, avec des professeurs de haut niveau, normaliens, agrégés, va poursuivre son exploration d'un enseignement $d u$ français sans latin, mais ne produira aucun outil rénové. Le fameux Mauger bleu, ne sera publié qu'en 1952, suivi en 1970 d'une autre méthode, d'inspiration audio-orale, Le Français et la vie.

La méthode directe est celle qui est largement recommandée et diffusée, au moins dans les colonies ${ }^{19}$, selon des modalités d'organisation interne qui peuvent varier, mais sans recherche véritable d'autres dispositifs d'apprentissage. Il s'agit d'exposer les élèves à une représentation du monde que l'on va apprendre à formuler en français.

Pour ce qui nous intéresse le plus directement ici, la référence à la nomenclature grammaticale de 1910 va avoir un effet stabilisateur sur les choix constatés, qu'il s'agisse des textes des programmes ou des méthodes en usage dans les différents cours pour adultes notamment ${ }^{20}$. Certes la linguistique, science encore nouvelle, pratiquée et diffusée dans un cercle restreint de chercheurs (voir Chevalier 2005), mais portée par un grand dynamisme créatif, n'exerce cependant aucune influence sur l'organisation des cours de français. Meillet, Tesnière, Guillaume, Bally, Vendryes, et autres, sont au travail, mais l'écho de leurs recherches ne s'opérera que bien après la deuxième guerre mondiale ${ }^{21}$.

Pour autant, dans le cadre de la deuxième grammaire scolaire, on procède à un certain nombre d'ajustements (voir Chervel $1977: 184$ et 230), autour des compléments d'objet direct et indirect, aux alentours de 1920, ainsi que du complément d'agent dont le principe est acquis vers 1930, mais des ajustements de détail, non une réorganisation du dispositif.

En revanche, on relèvera durant cette période la publication de quatre grandes sommes grammaticales, La pensée et la langue. Méthodes, principes et plan d'une théorie nouvelle du langage appliquée au français, de Ferdinand Brunot (1922), Des mots à la pensée. Essai de grammaire de la langue française, en 7 volumes (1911-1940), Le Bon usage, de Maurice Grévisse (1936), ainsi que La Grammaire Larousse du XXe siècle (1936), ouvrages aux orientations et aux bases épistémologiques fort différentes, mais qui témoignent d'une même volonté d'offrir la description la plus complète possible de la langue française, mais trois grammaires et non trois ouvrages de linguistique. Comme si la grammaire, par ces différentes sommes, voulait affirmer sa prééminence par rapport à une linguistique à venir.

\section{1945, un autre monde}

La Deuxième Guerre mondiale voit surgir un nouvel ordre du monde. Les empires coloniaux, français et anglais, sont appelés à disparaitre, ce qui va conduire à envisager une autre manière de penser le maintien et la diffusion des langues du colonisateur.

\subsection{Dans les colonies}

L'empire colonial laisse la place à l'Union française qui rassemble la France métropolitaine, les départements d'outre-mer, les territoires d'outre-mer et les états 
associés (27 septembre 1946). La France lâche du lest, alors que les premiers troubles éclatent en Indochine, en Algérie et à Madagascar. prend la place du Service des ÆEuvres françaises à l'étranger, créé en 1920, lequel faisait suite à la création du Bureau des écoles et des œuvres françaises à l'étranger en1909. Le Quai d'Orsay se dote d'un outil d'organisation d'une politique de diffusion et d'influence culturelles à l'étranger qui se traduira par la création de nombreux postes d'attachés culturels et progressivement par la diffusion d'outils d'enseignement du français.

Mais ces deux domaines apparemment distincts d'intervention de la France à l'étranger vont progressivement se rejoindre sous l'effet de l'Histoire et d'une évolution plus rapide que prévue. La France d'Outre-mer, c'est d'abord une France qui tente d'interrompre un processus de décolonisation qu'elle sent venir en absorbant l'espace colonial, désormais partie intégrante de cette France élargie à l'espace du monde. Tentative vaine qui n'empêchera nullement la décolonisation de s'opérer, mouvement qui fera apparaître à la grande surprise des acteurs un espace nouveau, la francophonie et aura pour effet induit d'engager un profond mouvement de rénovation de l'action culturelle à l'étranger. Deux exigences conjointes, donner à la francophonie un outil nouveau de diffusion et d'enseignement de la langue, permettre au français, dans l'étranger traditionnel de disposer d'un outil rénové dont le français élémentaire, puis 
français fondamental, constitueront » un outil de diffusion large et rapide de la langue française ».

\subsection{Vers le français fondamental}

Si le français fondamental ou français élémentaire voit le jour dans le courant des années 50, son principe, même si Georges Gougenheim n'y fait jamais allusion, avait été évoqué bien plus tôt. "Français réduit", sur le plan orthographique, à un moment évoqué par l'Alliance française, « langue spéciale " rêvée semble-t-il par Paul Bert lors de son séjour comme Résidant général au Tonkin, ou " français simplifié » en direction des tirailleurs sénégalais. Georges Gougenheim., peut-être par méconnaissance de ces tentatives, n'y fera jamais allusion ${ }^{22}$, préférant se référer au Basic english, comme exemple, à ne pas suivre d'ailleurs, pour établir une langue de large usage, sur la base d'un matériel langagier restreint.

51 Une commission française pour l'Unesco est constituée 1947, puis le SUREOM (Service des relations avec l'étranger et l'outre-mer), 1947-1960, installé au ministère de l'Éducation nationale

Une commission spéciale présidée par Marcel Abraham, inspecteur général de l'Instruction publique est créée au ministère de l'Éducation nationale en 1951. On y trouve, notons-le au passage, d'anciens responsables de l'enseignement dans les colonies, Albert Charton, inspecteur général, André Davesne, inspecteur d'académie de la Dordogne. La direction scientifique de l'entreprise est confiée à Georges Gougenheim ${ }^{23}$.

53 Nous ne développerons pas ici tout ce qui se rapporte à l'élaboration du français fondamental (voir DHFLES, 36, 2006), opération longue qui en gros va de 1951, date de l'installation de G. Gougnenheim comme responsable de l'entreprise, à 1964, date de la publication de l'ouvrage chez Didier, qui rend compte des résultats obtenus et des recommandations associées.

\subsection{La destination du français élémentaire}

La destination du français fondamental est triple :

accélérer la diffusion du français dans l'Union française ;

s'adresser aux travailleurs nord-africains qui viennent en France ;

à l'étranger, fournir aux auditeurs les éléments solides de connaissance du français.

Comme le rappellent les auteurs à différentes reprises, il s'agit de substituer à une élimination empirique des formes considérées comme trop complexes ou d'un faible intérêt pratique pour l'apprenant, un inventaire raisonné fondé sur une enquête statistique.

Conçu initialement pour s'adresser d'abord aux populations des colonies, devenues «Union française », le français élémentaire dans ses usages pour l'enseignement va s'adresser aussi aux travailleurs nord-africains présents en France, et ils sont à l'époque de plus en plus nombreux, tant la reconstruction de l'économie française a besoin de bras, mais aussi aux publics de ce qu'il est convenu d'appeler, dans la terminologie française, l'étranger traditionnel. C'est d'ailleurs dans ce dernier domaine que l'usage en sera le plus intense, à la différence des territoires des colonies qui considéreront avec la 
plus grande réserve un outil linguistique considéré comme n'offrant que des ressources langagières insuffisantes.

\subsection{Une grammaire issue du français fondamental}

60 commission pour l'etude de élémentaire, on extrait celles qui peuvent éclairer ce que pourrait être une grammaire de base du français :

La grammaire a été allégée des formes rares ou inexistantes dans la langue parlée : le passé simple de l'indicatif, le plus-que-parfait du subjonctif. Seuls parmi les pronoms relatifs, qui et que ont été conservés. On évite ainsi une syntaxe difficile même pour des personnes de langue française. On a taillé parmi les formes nombreuses et touffues de l'interrogation. Mais rien n'a été sacrifié de ce qui fait la structure vivante de la langue. Malgré les difficultés qu'ils présentent à l'enseignement, on a gardé deux temps essentiels du passé : l'imparfait et le passé composé. (Gougenheim $1954: 7$ )

61 La grammaire du français fondamental n'est donc pas une grammaire au sens classique du terme, c'est-à-dire le rassemblement d'un certain nombre de règles destinées à rendre compte de l'organisation et du fonctionnement de la langue française, mais une liste de prescriptions dégagées à partir de l'inventaire des mots considérés comme les plus fréquents et les plus utiles. En résulte le choix d'un certain nombre de constructions, celles considérées comme les plus nécessaires.

Sans entrer dans le détail d'une présentation de ces prescriptions, on ne manquera pas d'être frappé par le caractère traditionnel de la nomenclature retenue. Nulle révolution, nul bouleversement terminologique, alors même que la sous-commission de grammaire avait été présidée par Emile Benveniste, grand linguiste, spécialiste reconnu des langues européennes, l'un des rares linguistes, avec Aurélien Sauvageot à figurer dans la commission, loin du profil classique d'un grammairien tel Aristide Beslais ${ }^{24}$, directeur général de l'Enseignement primaire au ministère de l'Éducation nationale, latiniste et auteur de nombreux ouvrages de grammaire. Et pour autant la commission du FF s'en remet à des choix très traditionnels qui méritent d'être interprétés :

La linguistique, non encore appliquée, n'a pas dans les années 50 fait sa percée dans l'enseignement $d u$ français. Certes les linguistes commencent à être connus, ainsi que leurs travaux, mais ce sont les grammairiens qui, en la matière, constituent le groupe de référence et plus des grammairiens qui s'inscrivent dans la mouvance scolaire traditionnelle (grammaire pour l'orthographe et grammaire pour apprendre le latin) que les grammairiens « savants ».

Georges Gougenheim lui-même n'est pas un linguiste au sens rigoureux du terme ${ }^{25}$ et son équipe est constituée d'enseignants dont les références en matière de langue sont forcément marquées par les références grammaticales traditionnelles.

grammaire $\mathrm{du}$ français fondamental est avant tout un répertoire de recommandations ou de prescriptions et à ce titre ne vise pas à proposer une autre représentation grammaticale du français.

Ce répertoire, comme l'ensemble du projet du français fondamental, est centré sur les publics d'adultes et ne prend pas en compte, au moins initialement, les publics scolaires, enfants et adolescents, qui apprennent le français à la même époque. 

grammaire du FLM et grammaire du FLE. Derrière une nomenclature apparemment partagée s'engagent des logiques descriptives différentes, la grammaire du FF étant une « grammaire sur corpus » et non une grammaire générale qui embrasse l'ensemble des propriétés de la langue française. Grammaire qui se fonde sur un répertoire issu du français parlé, même si cet oral est un oral soutenu, proche très souvent du français écrit.

\section{Conclusion}

La question du choix des formes de la langue à laquelle sont exposés les élèves et celle de leur dénomination est loin d'être secondaire, car elle installe un rapport à la langue dont il est difficile de se défaire. En dépit des très nombreux travaux engagés pour appliquer à l'enseignement des langues les apports de la linguistique, à partir des années 60, ou pour s'inspirer de travaux issus d'autres modèles grammaticaux, grammaires du sens ou grammaires de l'énonciation dans les années 80 , l'étude des formes de la langue selon une terminologie traditionnelle semble encore l'emporter.

Ce conservatisme, souvent dénoncé, doit être recherché, au-delà d'un confort d'usage fondé sur le maintien d'une tradition à ce point répandue qu'elle finit par relever d'une évidence que l'on ne veut pas interroger, d'abord dans une situation historique. Lorsque la France décide de s'engager dans une politique de diffusion du français, elle le fait avec des moyens institutionnels et matériels qui seront de plus en plus importants, nous les avons brièvement signalés, à partir d'un centre qui confère ainsi une pleine légitimité aux choix qui sont les siens ${ }^{26}$. Faute de disposer d'un outillage grammatical adapté, la France va s'appuyer sur les fondements de la deuxième grammaire scolaire et en diffuser les principes et la terminologie dans l'ensemble des lieux où le français sera enseigné. Certes, dans le même temps, des grammaires sont élaborées dans des contextes allophones, en Pologne, en Espagne ou au Japon, pour ne citer ici que quelques pays dans lesquels l'approche de la grammaire du français a suscité des travaux portés par des enseignants allophones. Mais la France se plaçant dans une logique de l'offre à partir de ses propres catégories descriptives n'a jamais voulu, ou pu, s'engager dans de telles voies ${ }^{27}$.

\section{BIBLIOGRAPHIE}

Alliance israélite universelle (1903). Instructions générales pour les professeurs. Paris.

Alliance israélite universelle (1901-1904). Revue des écoles de l'Alliance israélite universelle.

Bulletin de l'Alliance française (1884-1900). Paris.

Bulletin de l'Alliance israélite universelle (1861-1914). Paris.

Documents pour l'histoire du français langue étrangère ou seconde, 58-59 | 2017 
BERMONT, Daniel, avec la collaboration de Caroline LECLERC (2008). Armand Colin, histoire d'un éditeur, de 1870 à nos jours. Paris : Armand Colin.

CHAUBET, François (2006). La Politique culturelle française et la diplomatie de la langue. L'Alliance française (1883-1940). Paris : L'Harmattan.

CHERVEL, André (1977). Et il fallut apprendre à écrire à tous les petits français. Histoire de la grammaire scolaire. Paris : Payot.

CHERVEL, André (2006). Histoire de l'enseignement du français du XVII ${ }^{\mathrm{e}}$ au XX ${ }^{\mathrm{e}}$ siècle. Paris : Retz.

CHERVEL, André (2006). « Pour une histoire comparée des disciplines du FLE et du FLM ». Le Langage et l'homme, 85-98.

CHEVALIER, Jean-Claude (2001). « Diffusion du français en Europe de l'Est : 1920-1939 ». In M.C. KokEscalle et F. Melka (éds.). Changements politiques et statuts des langues, Histoire et épistémologie. Amsterdam : Rodopi.

CHEVALIER, Jean-Claude (1945). Conférence africaine de Brazzaville, 30 janvier 1944-8 février 1944. Paris : Ministère des Colonies.

CHISS, Jean-Louis (1982). « Le structuralisme linguistique de Georges Gougenheim : la linguistique française entre la philologie et le modèle phonologique ». Lynx, 95-120.

CORTIER, Claude (1998). Institution de l'Alliance française et émergence de la francophonie. Politiques linguistiques et éducatives (1880-1914) ». Thèse. Lyon : Université de Lyon 2.

COSTE, Daniel (1989). « Aurélien Sauvageot (1897-1988). Un aspect de son œuvre : aux origines de l'élaboration du Français fondamental ». Études de linguistique appliquée, 75, 24-39.

COSTE, Daniel (1998). « Recherche universitaire et enseignement du français langue étrangère. À propos d'une rencontre de $1961 »$. Langue française, 117, 82-98.

FRESCO, Moïse (1895), Exercices gradués de langue française. Méthode pratique à l'usage des pays où l'on parle une autre langue que le français, édité par l'auteur. Paris.

GAMBLE, Harry (2010). « La crise de l'enseignement en Afrique occidentale française (1944-1950) ». Histoire de l'Éducation, 128, 129-162.

GOUGENHEIM, Georges (1954). « Caractères et destination du français élémentaire ». L'Éducation nationale, 25

Gougenheim, Georges, Michea René, Rivenc Paul, SAuvageot Aurélien (1964). L'Élaboration du français fondamental (1 ${ }^{\text {er }}$ degré). Étude sur l'établissement d'un vocabulaire et d'une grammaire de base. Paris : Didier.

KAHN, Gisèle (1990). « Un manuel pour l'enseignement du français aux militaires indigènes, 1927 ». Le français dans le monde. Recherches et Applications, août-sept., 97-103.

KASPI, André (dir.) (2010). Histoire de l'Alliance israélite universelle, de 1860 à nos jours. Paris : Armand Colin.

MARKovits, Rahul (2014). Civiliser l'Europe. Politiques du théâtre français au XVIII ${ }^{e}$ siècle. Paris : Fayard.

Ministère de l'Éducation nationale (1954). Le Français élémentaire. CNDP.

Mission laïque française. Revue de l'enseignement colonial, organe des écoles laïques françaises hors de France, 1904-1907, devenue Bulletin de la Mission laïque française - Revue de l'enseignement colonial, 1907-1914. 
MoLLIER, Jean-Yves (1993). « Le manuel scolaire et la bibliothèque du peuple ». Romantisme, 80, 79-93.

Nishiyama, Noriyuki (2001). «L'impérialisme linguistique de Pierre Foncin en Méditerranée ». Documents pour l'histoire du français langue étrangère ou seconde, 27, 35-48

NiSHIYAMA, Noriyuki (2005). «Cours de vacances de l'Alliance française sous la III ${ }^{e}$ République et genèse de la formation des maîtres en français ». Enseignement du français au Japon, 33, 19-34.

OMER, Danielle (2007). « Le premier manuel d'apprentissage de la lecture de Moïse Fresco, auteur et éditeur de livres scolaires en français à Istanbul (fin du XIX ${ }^{\mathrm{e}}$-milieu du XX $\mathrm{XX}^{\mathrm{e}}$ siècle) ». Documents pour l'histoire du français langue étrangère ou seconde [En ligne], 38/39.

SPAETH, Valérie (2001). « La création de l'Alliance israélite universelle ou la diffusion de la langue française dans le bassin méditerranée ». In M.C. Kok-Escalle et F. Melka (éds.). Changements politiques et statuts des langues. Histoire et épistémologie. Amsterdam : Rodopi, 103-118.

VERGNAUD, Jean (1980). « La genèse de la nomenclature de 1910 et ses enseignements ». Langue française, 47, 48-75.

VIGNER, Gérard (1999). «Le français en Afrique noire : aux origines de l'école publique indigène ». In André Petitjean et Jean-Marie Privat (dir.). Histoire de l'enseignement du français et textes officiels. Metz : Université de Metz, « Didactique des textes », 271-288.

VIGNER, Gérard (2015a). « Un exemple de contextualisation d'une grammaire scolaire du français dans le cadre d'un programme a destination des enfants indigènes d'Algérie (1890) » http:// www.univ-paris3.fr/medias/fichier/actes-colloque grac_1450368800137.pdf.

VIGNER, Gérard (2015b). « L'École normale Jules-Ferry, école normale de l'enseignement colonial : une formation pour apprendre à enseigner dans les colonies (1902-1912) ». Documents pour l'histoire du français langue étrangère ou seconde, 55, 57-82.

\section{NOTES}

1. L'œuvre des écoles d'Orient est créée à Paris par un groupe de catholiques libéraux regroupés autour du baron Augustin Cauchy, soucieuse de soutenir l'implantation et l'action des écoles congréganistes implantées dans l'empire ottoman. Son premier directeur est un jeune abbé, enseignant à la Sorbonne, qui deviendra rapidement une personnalité essentielle dans le développement d'un enseignement catholique hors de France, l'abbé Lavigerie (1825-1892).

2. Pierre Foncin secrétaire général de l'AF ne manque pas en effet de rappeler pour ce qui est de l'année 1889 : «La majeure partie de nos subventions a été accordée, après avis de la section du Levant, aux écoles françaises du Levant, catholiques pour la plupart, ou grecques, arméniennes, syriennes, israélites ou laïques. Nous avons servi ainsi la cause de l'Influence française ». (BAF 29bis 1889 : 8). La liste des écoles subventionnées (p. 16) fait apparaître une très large majorité d'écoles confessionnelles. Armand Colin ne manquera pas non plus de faire don d'un certain nombre de manuels aux écoles subventionnées par l'Alliance.

3. Voir Vergnaud 1980.

4. Nous ne traiterons pas ici de l'action de la Mission laïque française en la matière, soucieuse avant tout de proposer une alternative laïque aux écoles des missions.

5. Dans le même document (p. 40), on rappelle de façon très ferme: «L'analyse grammaticale doit être faite de vive voix, au tableau. Le maître ne fera jamais couvrir à des élèves des pages entières d'analyse grammaticale, travail fastidieux, à peu près stérile. Les exercices d'analyse logique sont inutiles et interdits. " 
6. Pour une présentation de Moïse Fresco et de ses différents travaux, voir Omer 2007.

7. Ce qui n'empêche nullement l'AF de poser la question de l'élaboration de manuels appropriés. À l'occasion de son congrès tenu les 5,6 et 7 août 1889 , dans la $4^{\mathrm{e}}$ section sera abordée la question suivante: «Livres d'enseignement français pour les écoles indigènes : comment devrait être composé un livre indiquant les connaissances que le maître français peut enseigner utilement aux indigènes dans les régions énumérées ci-dessous : Levant, Egypte, Sénégal, Madagascar, IndoChine, Océanie ?» (BAF 1889, $28: 75)$. La réponse sera progressivement apportée dans les débuts $\mathrm{du} \mathrm{XX}^{\mathrm{e}}$ siècle.

8. Il sera un membre particulièrement actif de la commission de propagande du département de la Seine et son action sera saluée en termes particulièrement élogieux par Pierre Foncin: «L'honneur du succès revient [...] à l'organisateur actif et modeste qui a frappé à la porte de chaque mairie, converti le maire, dressé la liste des notables de chaque arrondissement, recruté parmi eux un comité, convoqué ce comité, qui lui a fait connaître l'Alliance, l'a décidé à agir, l'a entraîné, qui a tout préparé pour les conférences, a répandu les programmes et les feuilles d'adhésion, qui, en un mot, sans bruit comme sans relâche, a tout mis en mouvement. Celui-là est bien à sa place à la tête de notre commission de la propagande, c'est M. Armand Colin. » (BAF, 15-18, 24 février 1887).

9. C'est dans le tournant des années 1870 , qu'ouvrent à l'initiative de leur créateur un certain nombre de maisons d'édition: Henry Vuibert, Fernand Nathan, Alexandre Hatier par exemple, Louis Hachette s'étant déjà engagé sur le marché bien des années auparavant, dès 1826, ainsi que Charles Delagrave dès 1865. Armand Colin y découvre d'ailleurs le métier d'éditeur.

10. Jeu de mots utilisés par les deux auteurs de la collection, Auguste Merlette (1823-1899) et André-Casimir Hauvion (mort en 1899), pour se donner un « nom de scène ».

11. Cette collection sera constamment remise à jour en fonction de l'évolution des programmes, nouveaux programmes de 1882 et publication de la circulaire de 1910 sur la nomenclature grammaticale.

12. Pour reprendre ici l'expression de Pierre Nora, dans ses Lieux de mémoire, celle d'« instituteur national » utilisée à propos d'Ernest Lavisse.

13. Pour une approche de la vie d'Armand Colin et du marché des livres scolaires dans les premiers temps de la III ${ }^{e}$ République, voir Mollier 1993 et Bermond 2008.

14. BAF, 13-14, septembre-novembre 1886, p. 133.

15. Nous retrouvons ici la question qu'avait posée A. Chervel: "Les grands succès de la grammaire du français ont-ils franchi nos frontières pour former des étrangers à la pratique de la langue française, Restaut, Wailly (et leurs Abrégés), Lhomond, Boniface, Noël et Chapsal, La Grammaire selon l'Académie, Poitevin, Larive et Fleury sont-ils un jour devenus, dans le texte français ou en traduction, des manuels de français langue étrangère?» (2009: 92). On peut répondre pour partie à cette question en rappelant que, dans la mesure où l'enseignement du français, entre 1850 et 1914 , dans les pays du Levant -- pour nous limiter ici à cette aire géographique --, prenait place dans un cadre scolaire, l'usage ou la référence à un manuel s'imposait, seuls les manuels français de grammaire étant disponible sur ce marché. Un inventaire des bons de commande établis par les écoles pourrait apporter un utile éclairage sur ce point.

16. Voir Vigner 1999.

17. Le Congrès de l'Éducation coloniale qui se tient à Paris en 1931 en même temps que l'Exposition coloniale, consacre sa rencontre au thème de l'adaptation de l'enseignement dans les colonies.

18. Voir « De l'École de préparation des professeurs de français à l'étranger à l'UFR DFLE. Histoire d'une institution (1920-2008)», sous la direction de M. Berré et D. Savatovsky, Documents pour l'histoire du français langue étrangère ou seconde, 44, 2010. 
19. Une exception est faite cependant pour les territoires de l'Indochine dans lesquels l'enseignement des langues telles que le vietnamien est maintenu ou celui de l'arabe dans des pays tels que la Tunisie, le Maroc ou du malgache à Madagascar.

20. Ainsi de la présentation de la nomenclature grammaticale présente dans la méthode France, $1^{\text {ère }}$ année de français, Camerlinck, 1919, Allyn et Bacon, Boston, qui significativement apporte les précisions suivantes: "The following terms are taken from the Arrêté du Ministre de l'Instruction publique et des Beaux- Arts, July 25, $1910 »$.

21. Il est difficile de situer le moment précis où la linguistique va exercer sur l'enseignement du français langue étrangère une influence déterminante (si tant est que cette influence ait été aussi déterminante que l'on serait tenté de le croire aujourd'hui). Mais l'excellent article de D. Coste (1998), « Recherche universitaire été enseignement du français langue étrangère. A propos d'une rencontre de 1961 », rend compte d'une rencontre organisée au CIEP de Sèvres, du 21 au 23 décembre 1961, mettant en relation le BEL (version première du BELC), sous l'auspice de la direction de la Coopération avec la Communauté et l'étranger au ministère de l'Éducation nationale. Les linguistes invités sont nombreux (citons notamment A. Culioli, G. Mounin, B. Pottier, A. Martinet, P. Léon, G. Gougenheim) et un programme est tracé qui doit permettre aux sciences du langage d'apporter leur contribution à un programme volontariste d'une action linguistique à l'étranger. Mais la prise en compte des sciences du langage dans l'élaboration des apprentissages prendra un certain temps et se situera plutôt dans le tournant des années 70 .

22. Voir Kahn 1990.

23. Pour une présentation des personnalités qui sont intervenues plus particulièrement dans l'élaboration du français fondamental, voir Coste 1988.

24. Qui par ailleurs publie en 1949, pour le compte du ministère de l'Éducation nationale, une nouvelle nomenclature, dite "Nomenclature Beslais » qui réactualise, sans la bouleverser, la nomenclature de 1910.

25. Georges Gougenheim publie en effet, en 1938, Le système grammatical de la langue française. Il s'efforce d'y regrouper les faits de syntaxe en système, et peut quelque part annoncer quelque part une approche structuraliste du français, il le fait malgré tout plus en grammairien qu'en linguiste de stricte obédience. Voir sur cette question Chiss 1982.

26. Centre qui comprend tout à la fois l'Éducation nationale avec le SUREOM et, plus tard, le CREDIF, installé à l'école normale supérieure de Saint-Cloud et le ministère des Affaires étrangères avec la Direction générale des relations culturelles et des œuvres françaises à l'étranger créée en avril 1945.

27. On notera cependant la création (récente, 2011) d'un groupe de recherche basé à Paris 3 (GRAmmaire et Contextualisation, le GRAC) qui se donne pour projet d'explorer cette voie.

\section{RÉSUMÉS}

Le projet colonial dans les débuts de la III $^{\mathrm{e}}$ République, comme la volonté de certaines élites françaises de réaffirmer la mission civilisatrice de la France, s'est traduite par une « offre de langue ", pour reprendre l'expression de l'historien François Chaubet, avec le souci de propager le plus largement possible la langue française. Cette propagation va passer par de multiples canaux et avoir pour effet induit de diffuser un appareil grammatical alors en pleine reconstruction dans l'espace scolaire français. Cet appareil, dont nous exposerons rapidement les sources, sera diffusé aussi bien par des instances privées, mais proches de l'État français, dans 
tous les secteurs de l'étranger, que par les autorités coloniales désireuses de mettre en place une école indigène. La grammaire, ossature de la langue, quelles que soient d'ailleurs les situations d'enseignement et d'usage, se trouve ainsi diffusée en différents endroits du monde, selon des répertoires métalinguistiques variés. Nous nous proposons de suivre ici, mais sans volonté d'exhaustivité, les cheminements d'un appareil qui selon les lieux d'usage, les publics et les époques, fera l'objet d'appropriations variées et de remaniements dont nous tenterons de cerner le profil

The colonial project in the early days of the Third Republic, as well as the will of certain French elites to reaffirm the civilizing mission of France, have resulted in a "language offer" in the words of the historian François Chaubet with the desire to spread as widely as possible the French language. This spread will go through multiple channels and resulted in particular in the dissemination of a grammatical framework which was then undergoing reconstruction in the French school space itself. The framework, whose sources we briefly caracterise, will be spread in all sectors abroad by both private entities - but close to the French State -, and by the colonial authorities committed to set up and maintain an indigenous school. Grammar, as backbone of the language, whatever the contexts of teaching and use, is distributed in different places of the world, under various metalinguistic directories. We intend to follow here, without pretention for exhaustiveness, the various paths of this grammatical framework, which, depending on the users, uses and areas, will undergo, different forms of appropriation and rearrangement, the profiles of which we shall try to identify

\section{INDEX}

Keywords : grammar in school, nomenclature, textbooks, elementary French

Mots-clés : Grammaire, scolaire, nomenclature, manuels, français élémentaire

\section{AUTEUR}

\section{GÉRARD VIGNER}

Éducation nationale / SIHFLES 